\title{
Molecular-level Antibody Repertoire Profiling and Engineering: Implications for Developing Next-generation Diagnostics, Therapeutics, and Vaccines
}

\author{
Jiwon Lee
}

Received: 16 February 2018 / Revised: 19 February 2018 / Accepted: 20 February 2018

(C) The Korean Society for Biotechnology and Bioengineering and Springer 2019

Through the application of quantitative analyses, design and optimization approaches, and technology developments to biological systems, chemical and biological engineers have made tremendous contributions to the fields of biotechnology, metabolic engineering, protein engineering, and synthetic biology. In particular, the successes in the field of industrial biotechnology focused on establishing a biobased economy where biomass is converted to generate chemical products, biomaterials, and biofuels highlight the progress of such integration. As a pioneer in the field, my father, Professor Sun Bok Lee has dedicated over 30 years of his research to the advancements of industrial biotechnology, and it is truly an honor to be a part of this special issue of Biotechnology and Bioprocess Engineering dedicated to commemorate his retirement.

Recently, our understanding of the human immune system, which consists of highly complex networks of immunological cells, molecules, and chemicals, has grown substantially over the years to the extent where one can take a more integrated and systemic approach to immunology. This development has now placed engineers at the forefront of a new and very rapidly growing interdisciplinary field of

Jiwon Lee ${ }^{*}$

Department of Chemical Engineering, The University of Texas at Austin, Austin, Texas 78712, USA

Department of Biological Chemistry and Molecular Pharmacology, Harvard Medical School, Boston, Massachusetts 02115, USA

Thayer School of Engineering, Dartmouth College, Hanover, New

Hampshire 03755, USA

Tel: +603-646-2674; Fax: +603-646-2277

E-mail: Jiwon.Lee@dartmouth.edu

This article is a summary of the talk given by Dr. Jiwon Lee on October 11, 2018 at the Special Symposium Honoring Professor Sun Bok Lee for His Retirement, Fall Meeting of the Korean Society of Biotechnology and Bioengineering, Seoul, Korea. immunoengineering, which is a research area that aims to apply engineering principles, standards, and tools to better comprehend our immune system, and then rely on the improved knowledge to guide the efforts to optimize various parameters of immune responses (Fig. 1A).

A principal component of the adaptive immunity is a diverse ensemble or repertoire of different antibody molecules, also known as immunoglobulin (Ig), that circulate in blood or coat mucosal surfaces and recognize a plethora of pathogenic molecules. These antibody molecules serve to protect the host against infectious agents and cancerous cells. In some instances, however, the production of self-reacting antibodies can trigger autoimmune diseases as well. Accordingly, health can be defined as a state where this balance is maintained and held steady, and perturbing the equilibrium within the system results in illness.

While antibodies were discovered over a century ago (the word "antibody" was coined in 1891 by Nobel laureate Paul Ehrlich), the molecular-level analysis of the antibody repertoire has been overlooked mainly due to the technical limitations. However, the recent technological advancements are now making it possible to dissect the diverse human antibody repertoires in various contexts of health and disease and provide a fuller depiction of the governing principles of how antibodies against infectious agents are generated while maintaining the aforementioned balance.

\section{Molecular-level Antibody Repertoire Profiling}

Antibodies are proteins secreted by a subset of B cells that recognize particular regions, or epitopes, within antigens ("antibody generator"). The basic structure of antibody 
A

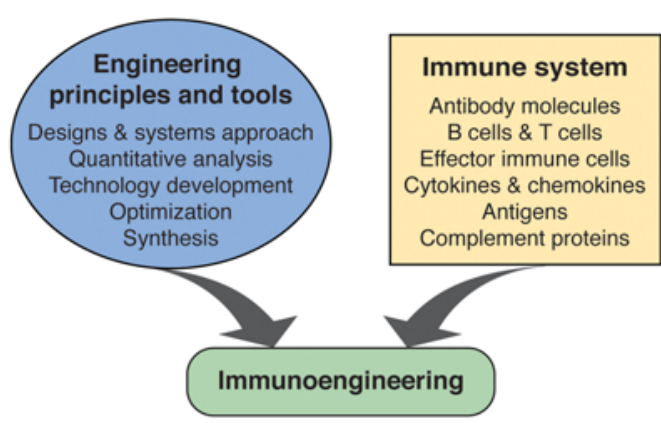

B

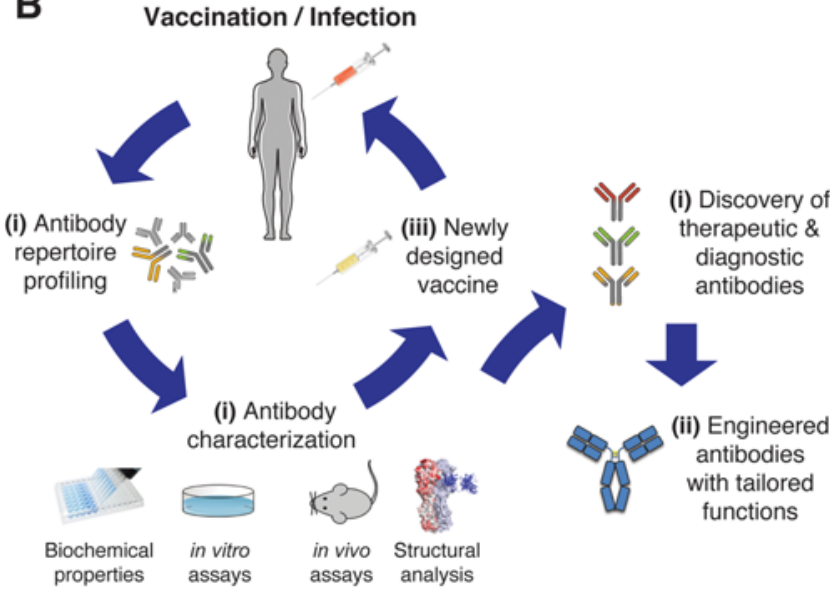

Fig. 1. Implications that molecular-level antibody repertoire profiling and engineering have for developing next-generation diagnostics, therapeutics, and vaccines. (A) Immunoengineering is an interdisciplinary research field where engineering principles and tools are applied to the immune system to optimize various parameters and generate the desired responses. (B) An illustration of how molecular-level antibody repertoire profiling can lead to the next-generation diagnostics and therapeutics with tailored functions, as well as newly designed vaccines that can elicit protective vaccine responses.

molecules (mainly determined from the work of the Nobel laureates Gerald Edelman and Rodney Porter) consists of two identical heavy chains and two identical light chains connected by disulfide bonds, with both heavy and light chains containing variable regions (called $\mathrm{VH}$ and VL, respectively) and constant domains. VH and VL, along with one constant domain from each of the heavy chain and the light chain comprise the antigen-binding fragment (Fab) while the rest is known as the crystallizable fragment $(\mathrm{Fc})$. While the Fab region mediates binding to cognate antigen, the $\mathrm{Fc}$ domain defines the antibody class (IgA, $\operatorname{IgD}$, IgE, IgG, or IgM).

The majority of contacts between Fab and its epitope occurs through the hypervariable regions within $\mathrm{VH}$ and VL termed the complementarity-determining regions (CDRs). The sequence variations in the CDRs arise from a random pairing of gene segments known as V(D)J recombination, as well as somatic hypermutations that occur upon being exposed to an antigen, resulting in antibodies with higher binding affinity and specificity (a process called affinity maturation). The resulting sequence diversity is fundamental to the generation of highly diverse antibody repertoires that have the capacity for binding an estimated to be at least $10^{12}$ different antigenic epitopes.

The repertoire of antibody molecules circulating in blood is the basis for the immune system to mount protective immunity against infectious agents, and it is shaped through various exposures to pathogens and vaccines encountered during one's lifetime. In the past, classic serology was the standard for examining the aggregate characteristics of serum antibodies (i.e., increase in abundance of antigenspecific antibodies following vaccination or infection), but it lacks any molecular-level information in regards to antibody compositions. Because Ig molecules are produced by $\mathrm{B}$ cells, however, their genetic information encoded by $\mathrm{B}$ cells can be examined as surrogates for Ig proteins. This approach was particularly revolutionized by the hybridoma technology developed by Nobel laureates César Milstein and Georges Köhler that enabled generation of monoclonal antibodies (mAbs), which refer to antibody produced by a single clone of cells, albeit at a low-throughput manner. The technology allowed the isolation of a large number of antigen-specific mAbs for the first time.

More recently, advances in Next-Generation Sequencing (NGS) technology with lowering cost and increasing throughput has completely transformed the field of antibody repertoire analysis, enabling the identification of antibody VH and VL sequences encoded by millions of B cells in exquisite detail. Since the seminal study published in 2009 reporting the first sequencing of antibody repertoire in zebrafish [1], numerous NGS-based studies have provided unprecedented insight into the dynamics of antibody repertoires in infectious disease, autoimmune disease, and cancer [2].

Yet, there remains a gap in our understanding of the antibodies circulating in blood at the protein level. This is because peripheral blood B cells represent only a small fraction of the total cells in the body that secrete antibody molecules, and accordingly, NGS of B cells does not provide quantitative information in regards to the antibody protein molecules in circulation. This gap is now slowly being filled through the emergence of bottom-up highresolution mass spectrometry (MS)-based proteomics as a powerful tool for identification, quantification, and characterization of antibody repertoire at the protein level [3]. Briefly, antibody molecules specific to particular antigens can be affinity-purified directly from blood of a human donor using immobilized antigen, and shotgun proteomic analysis of the eluate generates peptide mass 
spectra, whereby the sequences of these peptides are identified by searching against the donor-specific reference databases (this is because each person has unique antibody repertoire); if the matching sequence is not in the database, the peptide is not identified. Accordingly, the simultaneous improvements of NGS depth and MS mass resolution have enabled the molecular-level profiling of antibody molecules. The most prominent advantage of the molecular-level antibody profiling is that the analysis focuses primarily on the functionally relevant antibody molecules (i.e., abundantly present as protein molecules) directly isolated from the circulation.

Collectively, high-throughput antibody repertoire studies have successfully resulted in an explosion of discoveries of many pathogen-specific mAbs (e.g., HIV, MERS, influenza, tumor, etc.) identified directly from human samples. Subsequently, recombinant expression and detailed analysis of these $\mathrm{mAbs}$, ranging from biochemical properties (i.e., binding thermodynamics or kinetics), protective properties (i.e., in vitro and in vivo assays to measure the functionality against the target pathogens), and structural properties (i.e., identification of binding epitopes), have led to the accurate and clinically relevant insights regarding the precise features of mAbs that can provide effective protection and their protective mechanisms at an unprecedented details. Additionally, the pathogen-specific mAbs have great potentials as diagnostics and therapeutics (Fig. 1B, i).

\section{Molecular-level Antibody Engineering}

Antibodies can defend the host through multiple modes of action. In some instances, antibody binding may be sufficient to neutralize pathogens or their toxins directly. As an example, influenza virus binds to host cells via receptor-binding site (RBS) to initiate the infection cycle, and RBS-binding antibodies can sterically prevent the virus attachment. In other cases, antibody molecules rely on the recruitment of potent immune effector cells or immunological molecules to clear infected or cancerous cells, and this process is mediated by the binding between $\mathrm{Fc}$ domains and their target receptors, known as $\mathrm{Fc}$ receptors (FcRs), or complement proteins.

There are several different types of FcRs (each type binds to a different class of antibodies), and they are expressed on the surface of immune cells, which includes $\mathrm{B}$ cells, dendritic cells, natural killer cells, macrophages, and granulocytes. Because distinct types of immune cells express different groups of FcRs, it is this fine balance of binding affinity and specificity between Fc and FcRs, and expression profiles of FcRs on the target immune cells that ultimately determines the outcome of the immune effector functions [4]. Therefore, engineering of the Fc domain has significant implications on the functionality of therapeutic antibodies.

It is important to note that the optimization of antibody effector functions depends on detailed mechanistic understanding of protection mediated by individual pathogenspecific mAbs in the context of particular diseases. For antibodies capable of providing protection solely through binding, their Fab regions can be engineered to increase binding affinities and specificities [5]. However, for antibodies that mediate protection through effector functions, one needs to take a more refined approach because antibodies against different pathogens likely require distinct effector functions and/or immune cells for protection. Once the desired properties are identified, $\mathrm{Fc}$ can be engineered through various approaches, including amino acid substitution as well as glycoengineering [6].

Currently, more than $10 \mathrm{Fc}$-engineered antibodies are under clinical evaluation [7], which demonstrates the potential of fine-tuning the antibody functions beyond what nature has designed and optimize the potency of antibody molecules as therapeutics. Molecular-level engineering of mAbs has opened doors to design next-generation therapeutics precisely tailored to maximize the antibodies functions in the context of particular diseases based on understanding of how $\mathrm{Fc}$ impacts effector functions and dictates protection against infection (Fig. 1B, ii).

\section{Molecular-level Vaccine Engineering}

Vaccination remains as one of the most successful interventions to global human health. The principle of how vaccination works encompasses the administration of antigenic material derived from the pathogen to elicit antibodies to mount a successful defense against the infection. Classic vaccine designs are based on an empirical approach, and some vaccines have enjoyed great success, leading to eradication of infectious agents (i.e., polio, smallpox, etc.); however, traditional vaccine development has been insufficient for certain pathogens that can evade the immune response (i.e., HIV, hepatitis $\mathrm{C}$ virus, influenza, etc.).

The main reason why a vaccine fails to elicit protective responses is that only a small fraction of the generated antibodies bind to specific epitopes (called "protective epitopes") where the pathogen is vulnerable to antibodymediated functions. For viruses that mutate rapidly, they have evolved to conceal such protective epitopes through mutation and presentation of non-functional epitopes, diminishing the elicitation of antibody responses to these critical epitopes so they can evade the immune system. 
The next-generation vaccine engineering relies on an antibody-guided rational vaccine design approach aimed at focusing vaccine responses on the protective epitopes by minimizing non-protective epitopes [8]. The strategy mainly relies on the detailed molecular-level analysis of antibody repertoires linking the binding epitopes to protective functions, and, in particular, the high-resolution structural characterization of antibodies-antigen complexes has resulted in the understanding of the necessary molecular interactions required for protection.

Various protein engineering strategies, including removing or masking non-protective epitopes through excision of domains and glycan shielding, and conformational stabilization to expose the concealed protective epitopes, have been employed in various proof-of-principle studies, and they have demonstrated successful focusing of antibody responses $[9,10]$. Immunogens designed to elicit welldefined antibody responses are the basis for the nextgeneration vaccines that are tailored for individuals. Furthermore, the antibody repertoire profiling technologies can be utilized to measure the improved efficacy of the novel vaccines (Fig. 1B, iii).

\section{Concluding Remarks}

The first therapeutic use of antibodies can be traced back to 1890 when Emil von Behring and Kitasato Shibasaburo demonstrated the concept of passive immunization. Today, antibodies have achieved remarkable success as pharmaceuticals against numerous human diseases (approved antibodies generate a market value $>\$ 60$ billion annually), in particular, due to the tunable binding specificity and affinity of antibody molecules, which also make them highly valuable as diagnostics and research tools.

While vaccination remains as one of the most effective methods to prevent disease, the recent list of 10 threats to global health in 2019 listed by the World Health Organization includes vaccine hesitancy (https://www.who.int/emergencies/ ten-threats-to-global-health-in-2019), in part due to public misunderstanding of vaccine efficacy stemming from shortcomings of a very small number of vaccines. Because successful engineering of antibodies and vaccines hinges on our ability to examine the diversity and function of the antibody repertoire, this underscores the importance of molecular-level antibody repertoire analysis in the development of next-generation diagnostics, therapeutics, and prophylactics.

The distinctions between different disciplines are blurrier than ever, and the immunoengineering research field is becoming highly collaborative and multi-disciplinary. From the development of technologies and tools for analyzing massive sets of data to the application of engineering principles to develop more effective therapeutics and prophylactics, immunoengineering needs contributions from engineers equipped with in-depth knowledge of biology and immunology moving forward.

\section{Reference}

1. Weinstein, J. A., N. Jiang, R. A. White, 3rd, D. S. Fisher, and S. R. Quake (2009) High-throughput sequencing of the zebrafish antibody repertoire. Science. 324: 807-810.

2. Georgiou, G., G. C. Ippolito, J. Beausang, C. E. Busse, H. Wardemann, and S. R. Quake (2014) The promise and challenge of high-throughput sequencing of the antibody repertoire. Nat. Biotechnol. 32: 158-168.

3. Lee, J., D. R. Boutz, V. Chromikova, M. G. Joyce, C. Vollmers, K. Leung, A. P. Horton, B. J. DeKosky, C.-H. Lee, J. J. Lavinder, E. M. Murrin, C. Chrysostomou, K. H. Hoi, Y. Tsybovsky, P. V. Thomas, A. Druz, B. Zhang, Y. Zhang, L. Wang, W.-P. Kong, D. Park, L. I. Popova, C. L. Dekker, M. M. Davis, C. E. Carter, T. M. Ross, A. D. Ellington, P. C. Wilson, E. M. Marcotte, J. R. Mascola, G. C. Ippolito, F. Krammer, S. R. Quake, P. D. Kwong, and G. Georgiou (2016) Molecular-level analysis of the serum antibody repertoire in young adults before and after seasonal influenza vaccination. Nat. Med. 22: 1456-1464.

4. Park, H. I., H. W. Yoon, and S. T. Jung (2016) The highly evolvable antibody Fc domain. Trends. Biotechnol. 34: 895-908.

5. Xu, L., A. Pegu, E. Rao, N. Doria-Rose, J. Beninga, K. McKee, D. M. Lord, R. R. Wei, G. Deng, M. Louder, S. D. Schmidt, Z. Mankoff, L. Wu, M. Asokan, C. Beil, C. Lange, W. D. Leuschner, J. Kruip, R. Sendak, Y. Do Kwon, T. Zhou, X. Chen, R. T. Bailer, K. Wang, M. Choe, L. J. Tartaglia, D. H. Barouch, S. O’Dell, J.-P. Todd, D. R. Burton, M. Roederer, M. Connors, R. A. Koup, P. D. Kwong, Z.-y. Yang, J. R. Mascola, and G. J. Nabel (2017) Trispecific broadly neutralizing HIV antibodies mediate potent SHIV protection in macaques. Science. 358: 85-90.

6. Sondermann, P., and D. E. Szymkowski (2016) Harnessing Fc receptor biology in the design of therapeutic antibodies. Curr. Opin. Immunol. 40: 78-87.

7. Strohl, W. R. (2018) Current progress in innovative engineered antibodies. Protein Cell. 9: 86-120.

8. Lanzavecchia, A., A. Frühwirth, L. Perez, and D. Corti (2016) Antibody-guided vaccine design: identification of protective epitopes. Curr. Opin. Immunol. 41: 62-67.

9. Kwong, P. D., and J. R. Mascola (2018) HIV-1 Vaccines based on antibody identification, B cell ontogeny, and epitope structure. Immunity. 48: 855-871.

10. Burton, D. R. (2017) What are the most powerful immunogen design vaccine strategies? Reverse vaccinology 2.0 shows great promise. Cold Spring Harb. Perspect. Biol. 9: a030262.

Publisher's Note Springer Nature remains neutral with regard to jurisdictional claims in published maps and institutional affiliations. 